A Bremsstrahlung Spectrometer using k-edge and Differential Filters with Image plate dosimeters

C.D. Chen, A.J. Mackinnon, F.N. Beg, H. Chen, M.H. Key, J. A. King, A. Link, A.G. MacPhee, P.K. Patel, M. Porkolab, R.B. Stephens, L.D. VanWoerkom, K.U. Akli, R.R. Freeman

May 7, 2008

High Temperature Plasma Diagnostics 2008 Albuquerque, NM, United States May 11, 2008 through May 15, 2008 
This document was prepared as an account of work sponsored by an agency of the United States government. Neither the United States government nor Lawrence Livermore National Security, LLC, nor any of their employees makes any warranty, expressed or implied, or assumes any legal liability or responsibility for the accuracy, completeness, or usefulness of any information, apparatus, product, or process disclosed, or represents that its use would not infringe privately owned rights. Reference herein to any specific commercial product, process, or service by trade name, trademark, manufacturer, or otherwise does not necessarily constitute or imply its endorsement, recommendation, or favoring by the United States government or Lawrence Livermore National Security, LLC. The views and opinions of authors expressed herein do not necessarily state or reflect those of the United States government or Lawrence Livermore National Security, LLC, and shall not be used for advertising or product endorsement purposes. 


\title{
A Bremsstrahlung Spectrometer using k-edge and Differential Filters with Image Plate Dosimeters
}

\author{
C.D. Chen ${ }^{1}$, J.A. King ${ }^{2}$, M.H. Key ${ }^{3}$, K.U. Akli ${ }^{5}$, F.N. $\mathrm{Beg}^{2}$, H. Chen ${ }^{3}$, R.R. Freeman ${ }^{4}$, A. Link ${ }^{4}$, A.J. Mackinnon ${ }^{3}$, \\ A.G. MacPhee ${ }^{3}$, P.K. Patel ${ }^{3}$, M. Porkolab ${ }^{1}$, R.B. Stephens ${ }^{5}$, L.D. Van Woerkom ${ }^{4}$ \\ ${ }^{1}$ Plasma Science Fusion Center, Massachusetts Institute of Technology, Cambridge, MA \\ ${ }^{2}$ Department of Mechanical and Aerospace Engineering, University of California-San Diego, La Jolla, CA \\ ${ }^{3}$ Lawrence Livermore National Laboratory, Livermore, CA \\ ${ }^{4}$ College of Mathematical and Physical Sciences, The Ohio State University, Columbus, $\mathrm{OH}$ \\ ${ }^{5}$ General Atomics, San Diego, CA
}

\begin{abstract}
A Bremsstrahlung spectrometer using k-edge and differential filtering has been used with Image Plate dosimeters to measure the x-ray fluence from short-pulse laser/target interactions. An electron spectrometer in front of the Bremsstrahlung spectrometer deflects electrons from the x-ray line of sight and simultaneously measures the electron spectrum. The response functions were modeled with the Monte Carlo code Integrated Tiger Series 3.0 and the dosimeters calibrated with radioactive sources. Electron distributions with slope temperatures in the $\mathrm{MeV}$ range are inferred from the Bremsstrahlung spectra.
\end{abstract}

\section{Introduction}

In the fast ignition concept $t^{i}$, a picosecond-scale laser pulse is used to heat a compressed 300 g/cc DT plasma and ignite a hot spot in the fuel. The laser interacts with the preformed plasma near the relativistic critical surface and deposits its energy into hot electrons, which propagate to the core and deposit their energy. Hydrodynamic simulations have shown that 1-3 MeV electrons have the ideal range for efficient deposition in the hot spot ${ }^{\mathrm{ii}}$. The determination of laser-produced hot electron energy spectrum and conversion efficiency is therefore critical to assessing the feasibility of fast ignition.

The energy spectrum of hot electrons generated in the interaction of a short-pulse laser with a solid target has been previously measured or inferred using a variety of techniques, including electron spectrometers $^{\mathrm{iii}}{ }^{\text {iv }}$, nuclear activation ${ }^{\mathrm{v}}$, differentially filtered Bremsstrahlung spectrometers ${ }^{\mathrm{vi}}$, buried fluorescent foils $^{\text {vii }}$, proton emission spectra ${ }^{\text {viii }}$, and combinations of the above. These measurements are useful for measuring various components of the electron spectra. For the 1-3 MeV electrons of interest, however, Bremsstrahlung spectrometers are more useful; the vacuum electrons measured by electron 
spectrometers are severely affected by MV potentials, and nuclear activation is sensitive to energies higher than those of interest to fast ignition.

We report here on the adaptation of a Bremsstrahlung spectrometer that uses $Z$ and differential $\mathrm{Pb}$ filtering to determine the $\mathrm{x}$-ray spectrum up to an MeV. This paper will describe the design of the instrument, review the calibration of the spectrometer, and discuss the simulations and analysis techniques to determine the energy spectrum (usually quantified as the slope temperature) in the regime of interest.

\section{Spectrometer Design}

A diagram of the Bremsstrahlung spectrometer is shown in Figure 1. The spectrometer consists of 13 filters of increasing $\mathrm{Z}$ from $\mathrm{Al}$ to $\mathrm{Pb}$, and then increasing thicknesses of $\mathrm{Pb}$ for differential filtering. The filters are 1 inch square with a $250 \mu \mathrm{m}$ sheet of Mylar taped to both faces to minimize the contribution of secondary electrons $<150 \mathrm{keV}$. This design was primarily adapted from R. Nolte ${ }^{\mathrm{ix}}$, where Thermoluminescent Detectors (TLD's) held in PVC trays were used as dosimeters. In our design we use Fuji BAS-MS Image Plates. The image plates have higher sensitivity than the TLD's and their spatial resolution allows for verification of diagnostic alignment. The image plates and filters are stacked in a 6 $\mathrm{mm}$ thick interlocking Lexan cartridge loaded into a $1.8 \mathrm{~cm}$ thick $\mathrm{Pb}$ box which shields up to $2 \mathrm{MeV}$
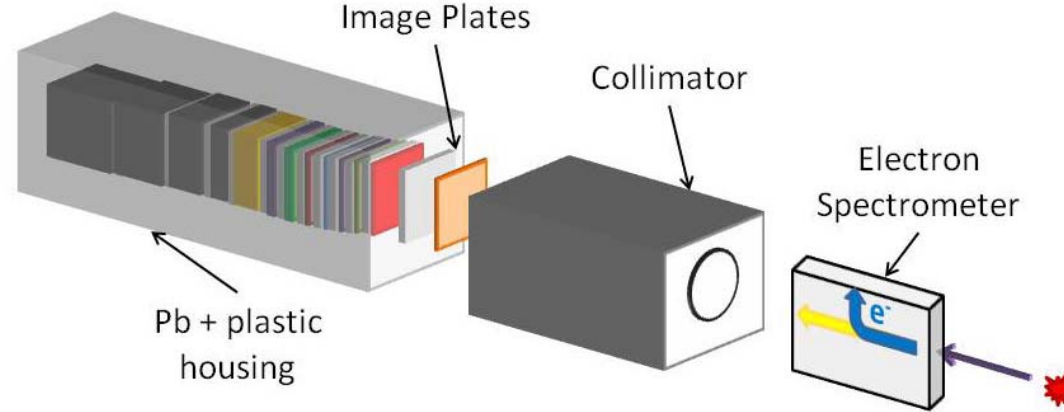

photons. The Lexan cartridge allows for rapid loading and unloading of spectrometer, is light-tight to preserve the image plate data, and reduces the noise from scattered

Figure 1. A diagram of the Bremsstrahlung spectrometer. The image plates are in a Lexan cartridge that fits into the $\mathrm{Pb}$ housing. The electron spectrometer deflects incident electrons. 
shielding. A $12.5 \mathrm{~cm}$ long $\mathrm{Pb}$ collimator with a $1 / 2$ " diameter hole is also used to reduce noise from fluorescence off the vacuum chamber walls. In our experiments strong electron beams that escape the target are also present along with the Bremsstrahlung emission. We use a modified electron spectrometer to deflect incident electrons and also to compare the vacuum electron spectra along the same line of sight as the Bremsstrahlung spectrometer. A $6 \mathrm{~mm}$ thick Teflon block is placed in front of the Bremsstrahlung spectrometer to stop any additional electrons.

\section{Dosimeter Calibration}

BAS-MS image plates use a $\mathrm{BaFBr}_{0.85} \mathrm{I}_{0.15}$ phosphor layer. When exposed to $\mathrm{x}$-rays, electrons in the phosphor layer are excited to a metastable state, where absorption of red light results in emission of blue light. The image plates are light and time sensitive, and good procedural controls are required for proper dosimetry. Following exposure, the image plates are kept in the light-tight Lexan cartridge and wrapped in a heavy cloth to prevent stray light from erasing the plates. They are scanned with a FLA7000 image plate scanner which reads out PhotoStimulated Luminescence values (PSL's) by exposing the plates with a red laser. Since image plates fade with time, the fade curve was measured from 5 to 50 minutes by exposing the image plates for $32 \mathrm{sec}$ to a filtered Cs-137 (662 keV) source. It was found that they fade about $20 \%$ in the first 30 minutes and then level off from 30 to 50 minutes. The image plates are thus scanned between 30 and 50 minutes after exposure on the flat part of the fade curve. We also assume in our analysis that the reading off the image plate is proportional to the total energy deposited in the active layer. To test this assumption and calibrate the plates, they were exposed to a Cd-109 source (22 keV) and a filtered Cs-137 source for 60 and 32 seconds, respectively, and scanned at exactly 30 minutes after the start of exposure. Using a 1-D monte carlo simulation from the Integrated Tiger Series $3.0^{\mathrm{x}}$ code package, the total energy deposited is compared to the PSL readout. The ITS code tracks electron and gamma ray showers, including such physics as elastic and Compton scattering, pair 
production, and $x$-ray fluorescence. The calibrations with the two sources were consistent, giving $1.47 \pm$

9\% MeV/PSL for the Cd-109 source and $1.24 \pm 15 \%$ for the Cs-137, where the larger error bar from the

Cs-137 exposure is from uncertainties in the activity of the source.

\section{Simulation of Response Matrices}

The response of the spectrometer to electrons incident on the target is broken down into 2

components. The Spectrometer

Response Matrix (SRM) is calculated in 1-

D using ITS and models the response of

the image plates to incident photons.

This response is shown in Figure 2. Each

of the 13 lines corresponds to the

response of the image plate behind the

different filters. The SRM is built up by

simulating the energy deposition in the

active layer of the 13 image plates in

response to 150 logarithmically spaced

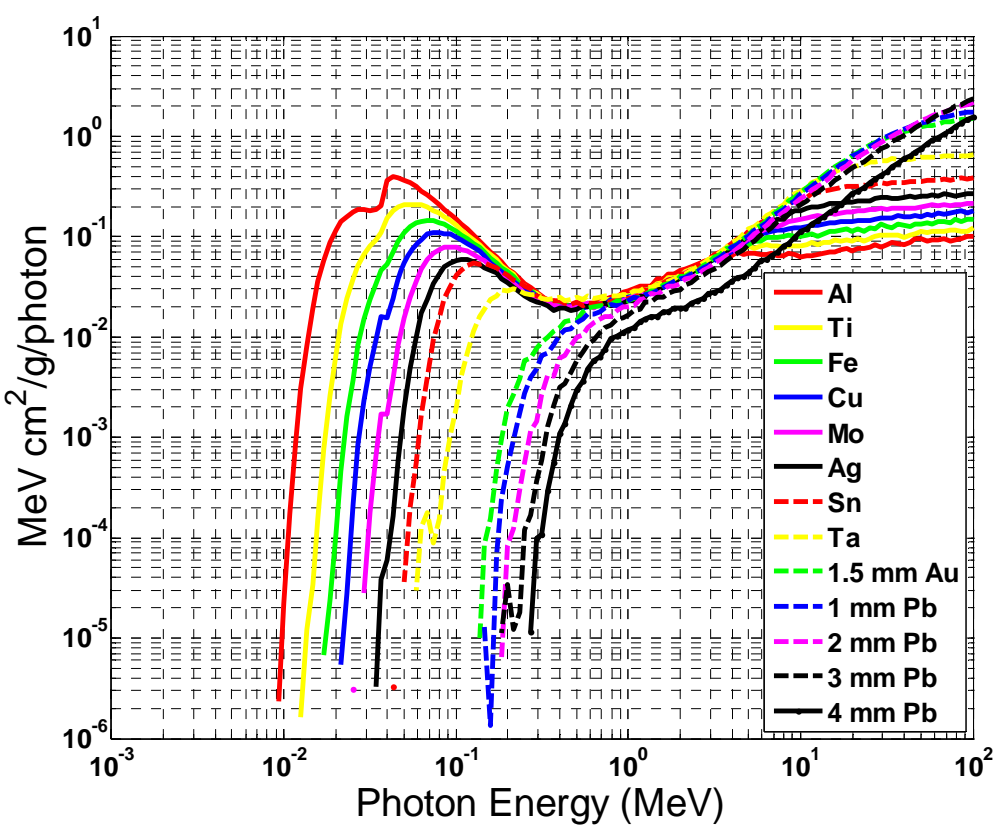

Figure 2. Spectrometer channel response (SRM) calculated from ITS 3.0. Each curve represents the energy deposited in that layer by the photon spectrum.

photon spectral bins from $1 \mathrm{keV}$ to $100 \mathrm{MeV}$. The second component, the Target Response Matrix (TRM), is modeled in 3-D with ITS for each target type. 80 logarithmically spaced electron spectral bins from $10 \mathrm{keV}$ to $100 \mathrm{MeV}$ are injected normally into a $30^{\circ}$ full cone angle from a $8 \mu \mathrm{m}$ diameter source.

The Bremsstrahlung spectrum into a $5^{\circ}$ cone angle off the rear surface is calculated for the photon emission. The SRM and the TRM are multiplied together to obtain the full response matrix for the electrons. 


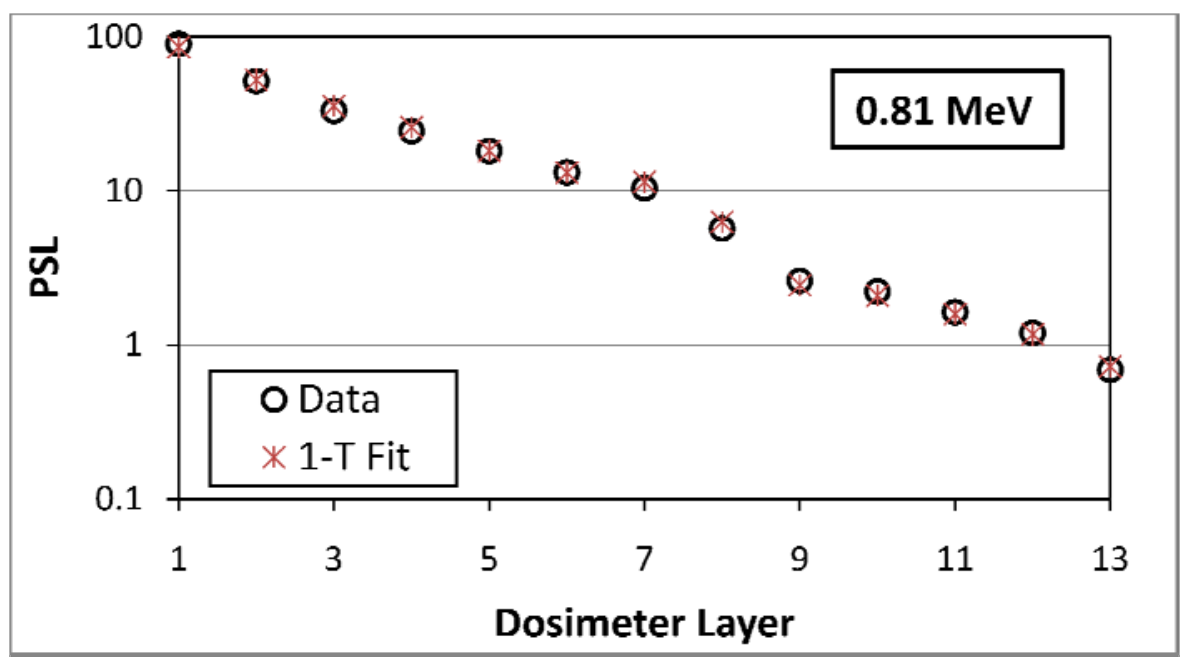

Figure 3. A 1-T Boltzmann distribution provides a good fit to the measured data under our experimental conditions.
The mean

deposition values from each of the 13 images plates are taken as the measured data. The electron spectra can be back calculated a number of ways, including fitting sample spectra and

maximum entropy techniques. For our experiments, we have found that a 1-temperature Boltzmann distribution of electrons provides a good fit to the measured data. Figure 3 shows the fit to a sample shot where an $\mathrm{Al} / \mathrm{Cu} / \mathrm{Al}$ sandwich target was irradiated with $57 \mathrm{~J}$ of $1.06 \mu \mathrm{m}$ light, $0.7 \mathrm{ps}$, on the TITAN laser at Lawrence Livermore National Laboratory. A Boltzmann distribution of electrons with a 0.81 $\mathrm{MeV}$ slope temperature provides a good fit to the data. Other distributions, such as a 1-D relativistic Maxwellian, also fit the data with a very similar slope temperature. The sensitivity of the measured distribution to spectral shape is still under investigation. The actual Bremsstrahlung spectrum can be calculated by multiplying the fitted spectra by the TRM. Alternatively, the x-ray spectra can be inferred using maximum entropy techniques from only the SRM.

This work was performed under the auspices of the U.S. Department of Energy by Lawrence Livermore National Laboratory under Contract DE-AC52-07NA27344.

'M. Tabak, et. al., Phys. Plasmas 1, 1626 (1994)

ii S. Atzeni, Phys. Plasmas 6, pp. 3316-3326, (1999)

iii G. Malka \& J. Miquel, Phys. Rev. Lett., 77, pp. 75-78, (1996)

${ }^{\text {iv }}$ K.A. Tanaka, et. al. Phys. Plasmas 7, pp. 2014-2022 (2000) 
${ }^{v}$ S.P. Hatchett, Phys. Plasmas 7, pp. 2076-2082 (2000)

vi P.A. Norreys, Phys. Plasmas 6, pp. 2150-2156 (1999)

${ }^{v i i}$ M. H. Key, et. al. Phys. Plasmas 5, pp. 1966-1971 (1998)

viii F.N. Beg, et. al. Phys. Plasmas 4, pp. 447-457 (1997)

${ }^{i x}$ R. Nolte, et. al., Radiat. Prot. Dosim. 84, pp. 367-370 (1999)

× J.A. Halbleib, R.P. Kensek, T.A. Mehlhorn, G.D. Valdez, S.M. Seltzer, and M.J. Berger, "ITS Version 3.0: The integrated TIGER Series of Coupled Electron/Photon Monte Carlo Transport Codes," Technical Report SAND911634, Sandia National Laboratories, 1992 